\title{
FENOLOGÍA Y PRODUCTIVIDAD PRIMARIA NETA AÉREA DE SISTEMAS PASTORILES DE Panicum maximun EN EL DPTO. MORENO, SANTIAGO DEL ESTERO, ARGENTINA, DERIVADA DEL NDVI MODIS
}

\section{PHENOLOGY AND ABOVEGROUND NET PRIMARY PRODUCTIVITY OF Panicum maximum PASTORALIST SYSTEMS IN THE DEPARTMENT OF MORENO, SANTIAGO DEL ESTERO, ARGENTINA, DERIVED FROM MODIS NDVI}

\author{
José Luis Tiedemann ${ }^{1}$
}

\begin{abstract}
Resumen
Los parámetros fenológicos y la productividad primaria neta aérea (PPNA) de los sistemas pastoriles de Panicum maximun en el Dpto. Moreno, Santiago del Estero, Argentina, en el período 2008-2014, fueron determinados a partir de series temporales de NDVI MODIS. El Inicio, Punto Medio y Fin de las estaciones de crecimiento tuvieron mayor frecuencia, respectivamente, en enero, marzo y junio. Se encontraron diferencias significativas $(p<0.01)$ en la Amplitud, Base y PPNA entre estaciones de crecimiento debido a anomalías en la precipitación media estacional. Las estaciones de crecimiento con mayor Longitud y Amplitud tuvieron mayor PPNA. Se encontraron significativas relaciones directas entre la Amplitud y la PPNA ( $\left.r^{2} 0.50\right)$. No se encontraron relaciones significativas entre la Base y la PPNA ( $p>0.05)$. La PPNA en las estaciones de crecimiento con anomalías negativas de precipitación fue: $1790.2 \mathrm{~kg}$ ms ha (20082009) y $3085.22 \mathrm{~kg} \mathrm{~ms}$ ha (2012-2013). La PPNA en las estaciones de crecimiento con anomalías positivas de precipitación fue: $9245.23 \mathrm{~kg} \mathrm{~ms}$ ha (2009-2010); 11562.31 kg ms ha (2010-2011); $10514.00 \mathrm{~kg} m \mathrm{ma}$ (2011-2012) y $9528.51 \mathrm{~kg} \mathrm{~ms}$ ha (2013-2014). La PPNA de las estaciones de crecimiento con sequía fue un 78,7 \% menor que la PPNA de las estaciones de crecimiento con anomalías positivas de precipitación media. Las series temporales de NDVI MODIS son una invalorable herramienta para determinar la receptividad de los sistemas pastoriles y para prevenir y/o mitigar adversidades climáticas extremas como sequías.

Palabras clave: NDVI; fenología; sequía; productividad primaria neta aérea; Panicum maximun.
\end{abstract}

\begin{abstract}
Phenological parameters and net primary productivity of Panicum maximum pastoralist systems in the Department of Moreno, Santiago del Estero, Argentina, in the period 2008-2014 were determined from MODIS NDVI time series. The start, midpoint and end of growing seasons had higher frequency, respectively, in January, March and June. Significant differences $(p<0.01)$ in the Amplitude, Base and aboveground net primary production (ANPP) between growing seasons due to anomalies in seasonal rainfall were found. Growing seasons with high length and amplitude had higher ANPP. Significant direct relationship between the Amplitude and ANPP were found $\left(r^{2} 0.50\right)$. However, relationships between the Base and ANPP $(p>0.05)$ were not found. ANPP in growing seasons with negative anomalies of rainfall was: $1790.2 \mathrm{~kg} \mathrm{dm} \mathrm{ha-1}$ (2008-2009) and $3085.22 \mathrm{~kg} \mathrm{dm} \mathrm{ha}^{-1}$ (2012-2013). In growing seasons with positive anomalies of rainfall, ANPP was: $9245.23 \mathrm{~kg} \mathrm{dm} \mathrm{ha}^{-1}$ (2009-2010); $11562.31 \mathrm{~kg} \mathrm{dm} \mathrm{ha}^{-1}$ (2010-2011), 10514.00 $\mathrm{kg} \mathrm{dm} \mathrm{ha}^{-1}$ (2011-2012) and $9528.51 \mathrm{~kg} \mathrm{dm} \mathrm{ha}^{-1}$ (2013-2014). ANPP growing seasons with drought was $78.7 \%$ lower than ANPP growing seasons with positive anomalies of average rainfall. The MODIS NDVI time series are an invaluable tool for determining the receptivity of pastoral systems and for preventing and / or mitigate adverse weather extremes such as droughts.

Key words: NDVI; phenology; drought; aboveground net primary productivity; Panicum maximum.
\end{abstract}

\section{Introducción.}

La principal actividad ganadera en el Dpto. Moreno de la Provincia de Santiago del Estero
(Argentina), es la cría bovina extensiva, siendo la vegetación natural (Bosque Chaqueño y sabanas) la base de su alimentación (Pérez, 1992). La vegetación 
natural sufre procesos de degradación desde fines del siglo XIX (Morello \& Saravia Toledo, 1959). La tala excesiva del bosque, el sobrepastoreo y los cambios en el régimen de fuegos, degradaron al bosque y lo transformaron en superficies de arbustos densos y espinosos, o en bosque secundario con alta densidad de individuos (Boletta et al., 2006; Kunst et al., 2012). Este tipo de cobertura y estructura redujo significativamente la oferta de forraje herbáceo limitando severamente la receptividad y por ende la actividad ganadera (Abril \& Bucher, 2001; Kunst et al., 2003; Smit, 2005; Kunst et al., 2006; Kunst et al., 2012). Con el fin de revertir la situación, el Instituto Nacional de Tecnología Agropecuaria (INTA) introdujo germoplasma forrajero subtropical tales como Panicum maximun, Chloris gayana y Cenchrus ciliaris (Perez, 1992).

La intensificación de la ganadería en Santiago del Estero, se basó en la eliminación del estrato arbustivo y arbóreo menor a $3 \mathrm{~m}$ de altura del bosque Chaqueño (Figura 1, arriba) mediante rolados (roller chopping) y en la implantación de gramíneas megatérmicas como Panicum maximun (Kunst et al., 2003; Anriquez et al., 2005; Ledesma, 2006; Kunst et al., 2012), dando como resultado un parque compuesto por leñosas y pasto (Kunst et al., 2003) (Figura 1, abajo). Ambas prácticas simultáneas generan incrementos superiores al $100 \%$ en la productividad de materia seca de las gramíneas (Kunst et al., 2003; Kunst et al., 2012) o productividad primaria neta (Field et al., 1995; Fensholt et al., 2006).

La productividad primaria neta terrestre (PPN), es la integración temporal del incremento positivo de biomasa de la vegetación terrestre por unidad de superficie y tiempo (Field et al., 1995; Fensholt et al., 2003; Fensholt et al., 2006). Representa el nuevo carbono fijado como biomasa en tallos, hojas y raíces de todas las plantas en el ecosistema (Fensholt et al., 2003; Fensholt et al., 2006). La ausencia o presencia de vegetación con actividad fotosintéticamente activa tiene un dramático efecto a nivel regional y global (White et al., 1997), en parte, por la elevada influencia de la fenología foliar en el intercambio de energía y dióxido de carbono entre la biosfera y la atmósfera (Jolly et al., 2005; Arora \& Boer, 2005) y porque delimita el inicio y el fin de la estación de crecimiento y por ende su longitud (Field et al., 1995).

La longitud de la estación de crecimiento se relaciona directamente con la PPN o productividad de la vegetación estacionalmente activa (Field et al., 1995; White et al., 1997; Reed \& Brown, 2005; Arora \& Boer, 2005; Jönsson \& Eklundh, 2006; Chandola et al., 2010). Por cuanto la determinación los parámetros fenológicos contribuye a la correcta determinación de la PPN (Bradley et al., 2007).

La estrecha relación lineal existente entre el Índice Verde Normalizado (NDVI) y la fracción de radiación fotosintéticamente activa absorbida (Asrar et al.,
1984; Goetz \& Prince, 1996) posibilitó el uso de los Índices de Vegetación como potenciales indicadores de la productividad fotosintética a escala global y de paisaje (Nouvellon et al., 2000). La relación entre la PPN aérea (PPNA) y el NDVI se realizó a través del modelo empírico de Monteith (1977) basado en la eficiencia en el uso de la radiación (Nouvellon et al., 2000; Felsholt et al., 2003). Al respecto, fueron desarrollados una gran cantidad de trabajos, como el de Field et al. (1995) quienes propusieron un simple modelo de PPN global, combinando principios ecológicos con datos derivados de satélites para estimar la productividad global. Los autores se basaron en la amplia relación existente entre los principios ecológicos, los recursos climáticos, las características de las especies y la PPN. A su vez, Goetz et al. (1999) mediante un modelo de eficiencia de productividad conducido a partir de observaciones satelitales, generaron mapas de productividad primaria bruta y neta, respiración autótrofa, biomasa y otras variables biofísicas del bosque Boreal de Canadá. Hicke et al. (2002) mediante una serie temporal de NDVI de diecisiete años y un modelo de ciclo de carbono exploraron los cambios en la PPN en Norte América, encontrando pequeños pero significativos incrementos en la PPN en el período analizado.

Entre las fases fenológicas de la superficie de la tierra que pueden ser detectadas a través de perfiles de Índices de Vegetación están el inicio y el fin de la estación de crecimiento y por ende su longitud (Reed et al., 2003; Reed \& Brown, 2005; Chandola et al., 2010; de Beurs \& Henebry, 2010; Tan et al., 2011; Atkinson et al., 2012).

Los sistemas pastoriles de Panicum maximun predominantes en el Dpto. Moreno, Santiago del Estero, son importantes desde el punto de vista ganadero debido a que sustentan 268.000 cabezas de bovinos (Argentina, 2013) y desde un punto de vista ecológico como fuente de biodiversidad y diversificación del paisaje rural (Di Bella et al., 2004) y como sumidero de carbono (Rigge et al., 2013).

La disponibilidad de información de los parámetros fenológicos y productividad de los sistemas pastoriles es vital en zonas semiáridas, en especial cuando la región es afectada por adversidades climáticas extremas como sequías.

Con el fin de aportar información local sobre la dinámica estacional y la respuesta ante estrés ambiental de los sistemas pastoriles del Dpto. Moreno, Santiago del Estero, Argentina, los objetivos de este trabajo fueron: determinar los parámetros fenológicos (inicio, punto medio, fin, amplitud y base), modelar las estaciones de crecimiento, cuantificar la productividad primaria neta aérea de los sistemas pastoriles de Panicum maximun y analizar su respuesta ante anomalías de la precipitación media, en el período 2008-2014. 


\section{Materiales y métodos.}

La vegetación natural predominante en la Provincia de Santiago del Estero es el bosque Chaqueño semiárido, xerófito y abierto (Figura 1, arriba). La comunidad clímax o estrato superior es el bosque de Schinopsis quebracho colorado y Aspidosperma quebracho blanco (SAyDS, 2004). Presenta un estrato medio, conformado por especies de los géneros Zizyphus, Cercidium, Celtis, Caesalpinea y Prosopis entre otras (SAyDS, 2004). El estrato arbustivo está conformado por especies pertenecientes a los géneros Acacia, Mimosa, Geoffroea, Prosopis, Atamisquea, entre otras (SAyDS, 2004). Finalmente el estrato herbáceo está formado por gramíneas de los géneros Setaria, Gouinia, Digitaria, Eragrostis, Gymnopogon, Panicum, Heteropogon, Trichloris, Chloris, y dicotiledóneas como Ruellia, Justicia, Holocheilus, Trixis, Hyptis, entre otras (SAyDS, 2007).

En la Figura 1 (abajo) se puede observar el sistema pastoril como resultado de rolado de alta intensidad y siembra simultánea de Panicum maximun (Anriquez et al., 2005; Kunst et al., 2008). El Panicum maximun cv Gatton panic es la especie de mayor difusión, adaptación y persistencia debido a las características edáficas y climáticas imperantes en la zona (Renolfi et al., 1992; de León, 2004).

El área de estudio se centra en el Dpto. Moreno, ubicado en el centro este de la provincia de Santiago del Estero, entre los paralelos $26^{\circ} 53^{\prime}$ y $27^{\circ} 48^{\prime}$ de

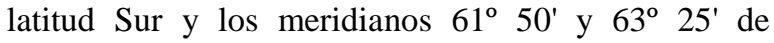
longitud Oeste (Figura 2, izquierda). La estación lluviosa comienza en Diciembre y se extiende hasta Marzo la precipitación media anual es de $750 \mathrm{~mm}$. La temperatura media mensual en Enero es $26.9^{\circ} \mathrm{C}$ (verano), y $12,4^{\circ} \mathrm{C}$ en Julio (invierno) (Boletta et al., 2006).

Los sistemas pastoriles (Figura 2) fueron identificados y seleccionados en terreno (Bradley et al., 2007). La verificación satelital de estas áreas de muestreo se realizó mediante Google Earth, una vez realizada se generaron polígonos vectores de las
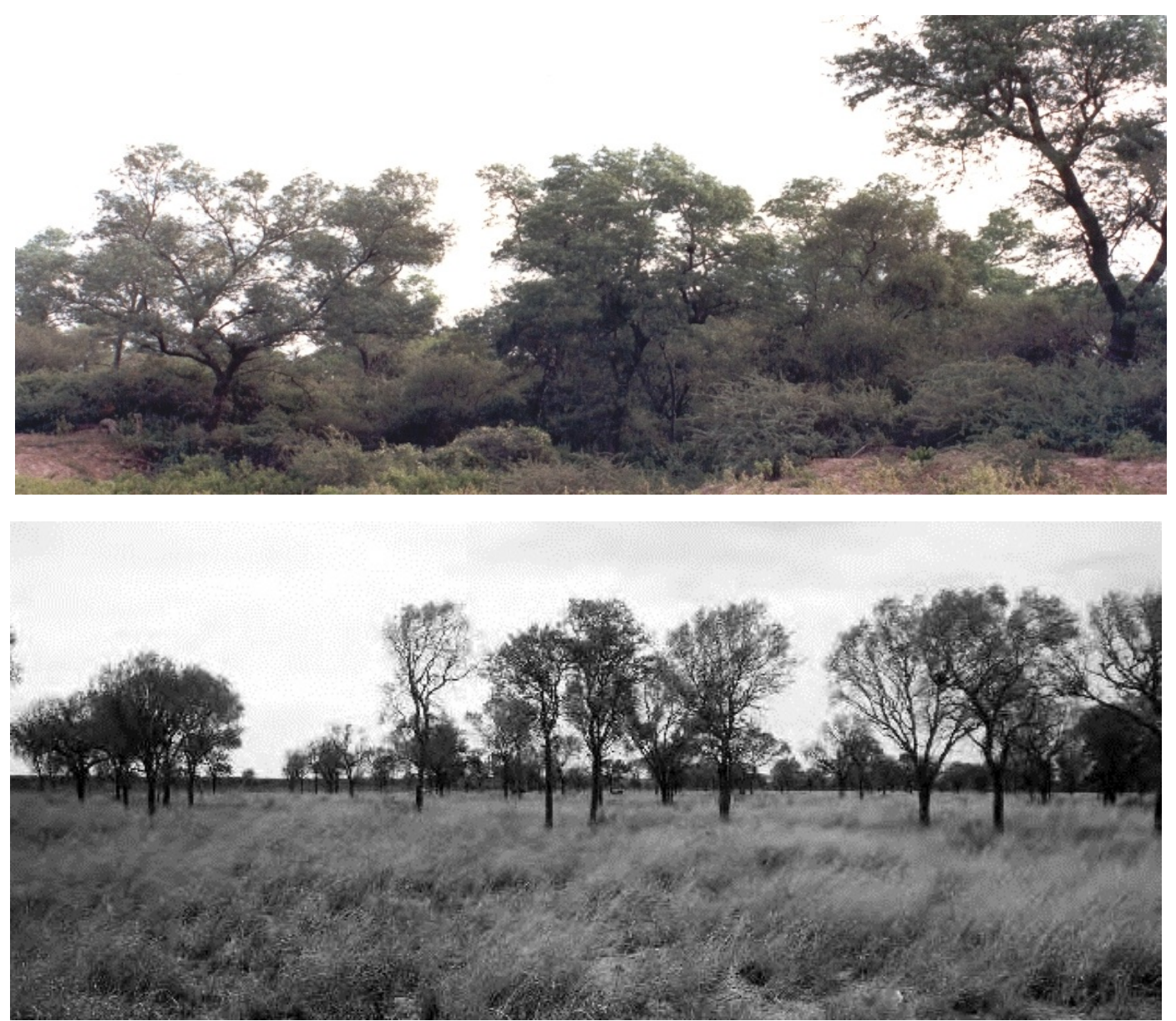

Figura 1. Arriba: Perfil típico del Bosque Chaqueño semiárido (Fuente: Zerda H. R. INPROVE-FCF-UNSE). Abajo: Perfil típico de Sistema Pastoril de Panicum maximum (Fuente: Kunst et al., 2008). 
mismas (Figura 2, derecha). Las superficies y coordenadas centrales de los sistemas pastoriles (SP) son: $\mathrm{SP}_{1}=1429$ has $(-62.70 \mathrm{~S} ;-27.25 \mathrm{O}) ; \mathrm{SP}_{2}=2866$ has $(-62.28 \mathrm{~S}$; $-27.67 \mathrm{O}) ; \mathrm{SP}_{3}=435$ has $(-62.25 \mathrm{~S}$; $27.43 \mathrm{O})$ y $\mathrm{SP}_{4}=2055$ has $(-62.84 \mathrm{~S} ;-26.98 \mathrm{O})$ (Figura 2, derecha).

La variable utilizada fue el Índice Verde Normalizado desarrollado por Rouse et al. (1973), y ampliamente conocido por sus siglas en ingles NDVI. El NDVI fue confeccionado mediante la siguiente ecuación NDVI $=(N I R-R E D) /(N I R+R E D)$, a partir de datos de reflectancia superficial MOD09GQ (TERRA AM) y MYD09GQ (AQUA PM) derivados del sensor MODIS (Moderate Resolution Imaging Spectroradiometer). Los productos utilizados tienen una resolución temporal diaria y una resolución espacial de 250m x 250m (Becker-Reshef et al., 2010). Los valores del NDVI oscilan en el rango de 1.0 y 1.0. Los pixeles con valores iguales o inferiores a 0.01 fueron enmascarados, ya que éstos se relacionan con cuerpos de agua, suelos salinos y suelo desnudo. Todos los datos de reflectancia superficial utilizados en este trabajo pertenecen al proyecto Earth Observing Systems - NASA, obtenidos en http://modis.gsfc.nasa.gov/.

El gran potencial de las series temporales de NDVI es obstaculizado por problemas instrumentales, cambios en el ángulo del sensor, variabilidad atmosférica (nubes, neblina, aerosoles y polvo), reflectancia en dos direcciones de las coberturas de la tierra, que alteran y generan valores anómalos de NDVI (Bradley et al., 2007; Tan et al., 2008; Atkinson et al., 2012). Los mapas de la serie temporal fueron rigurosamente controlados. El primer control se realizó mediante el módulo EOSDIS Worldview alpha con imágenes Aqua \& Terra de reflectancia corregida. El segundo control fue realizado sobre los mapas de NDVI, mediante el módulo de animación Media Viewer (Idrisi Taiga) con secuencias de cinco segundos de duración para cada mapa (Eastman,

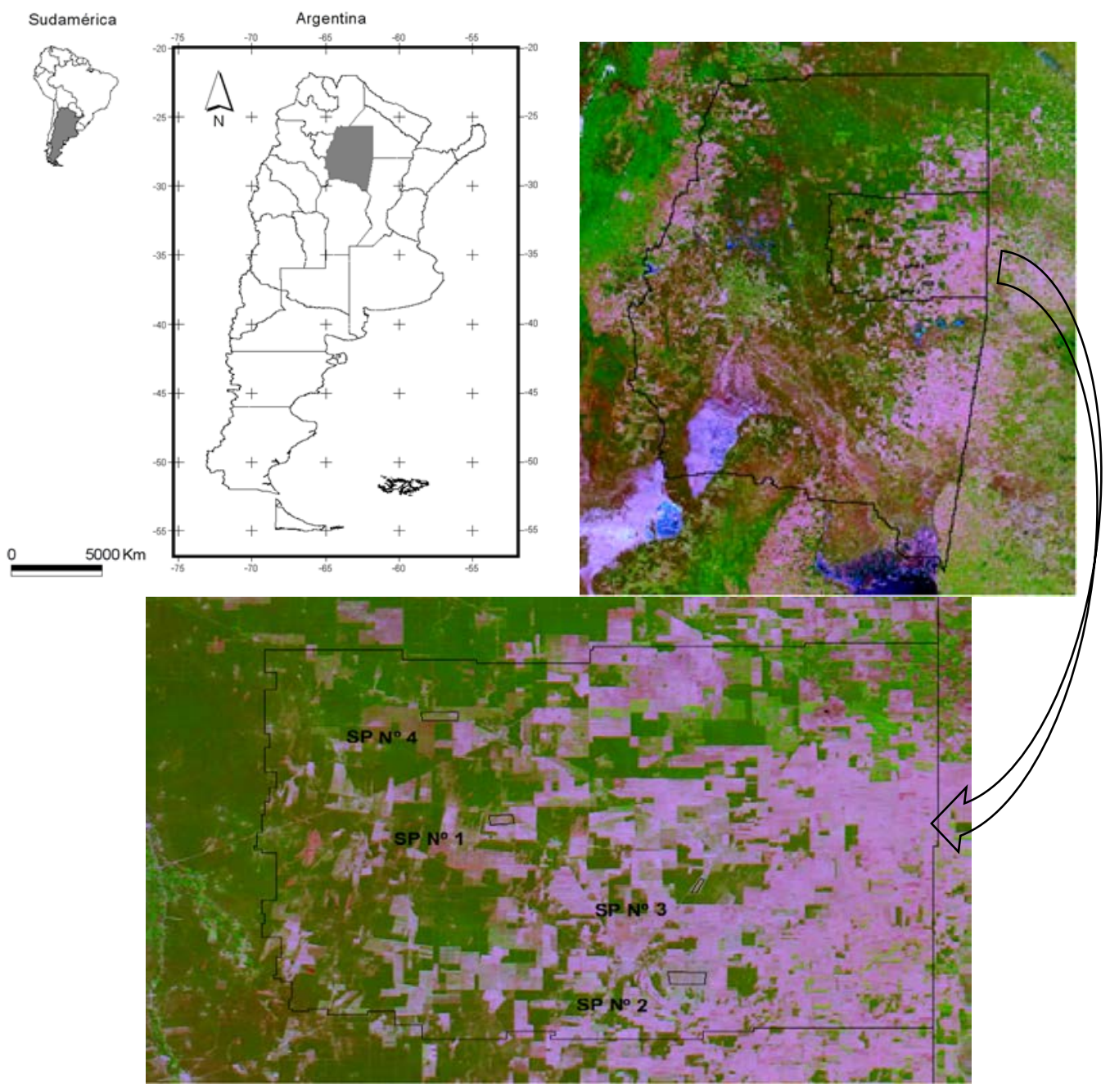

Figura 2. (Izquierda) Argentina en Sudamérica y Santiago del Estero en Argentina; (Derecha) Santiago del Estero y el Dpto. Moreno (polígono en centro este). (Abajo) El Dpto. Moreno y áeas de muestreo de los sistemas pastoriles (SP). 


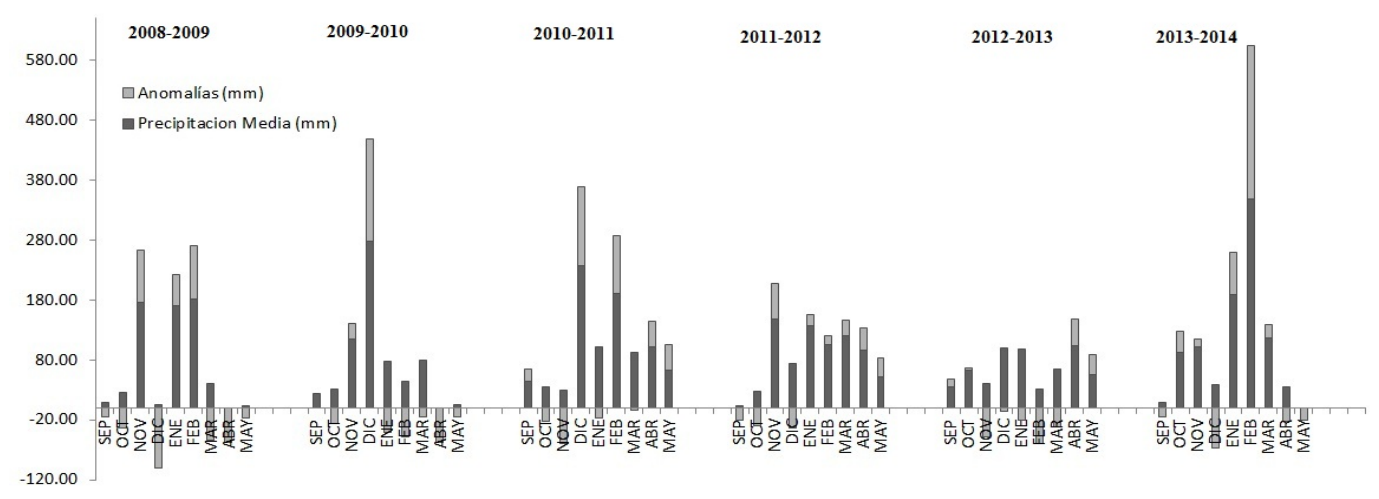

Figura 3. (Barras gris oscuro) Precipitación media mensual del período 2008-2014 y (barras gris claro) Las anomalías de precipitación media mensual con respecto al valor normal histórico (1918-2014). (Fuente: Sociedad Rural del Noreste Santiagueño).

2009). Fueron eliminados, manualmente, los mapas con contaminación residual de nubes y con fallas en la calibración de los sensores (Ho et al., 2011), quedando conformada una serie temporal de 291 mapas de NDVI MODIS que se extendió de agosto de 2008 hasta agosto de 2014.

Los valores medios de NDVI de los SP fueron extraídos de la serie temporal mediante el módulo TSF (Eastman et al., 1995). Se minimizó el ruido residual de la serie temporal del NDVI, mediante una técnica de suavizado tipo media móvil (Reed \& Brown, 2005; Balzarini et al., 2008; Tan et al., 2008; Atkinson et al., 2012). Se generó una gráfica simultánea de las series de observaciones originales y suavizadas en función de la variable regresora (tiempo) para corroborar que la técnica de suavizado no haya provisto una señal falsa (Balzarini et al., 2008). La media móvil de la serie temporal y los análisis estadísticos fueron realizados con el software Infostat (Di Rienzo et al., 2008).

Para determinar las estaciones de crecimiento (EC) de los SP se utilizó un modelo de regresión cuadrática (de Beurs \& Henebry, 2005; de Beurs \& Henebry, 2010; Brown et al., 2012):

$\mathrm{EC}_{\mathrm{SP}}=\alpha \mathrm{NDVI}^{2}+\beta \mathrm{NDVI}+\gamma$

En donde la variable independiente es la serie temporal del NDVI (tiempo), $\alpha$ el parámetro cuadrático, $\beta$ la pendiente de la curva y, $\gamma$ la ordenada al origen que corresponde al NDVI en el inicio de la estación de crecimiento. La pendiente de la curva $\beta$ y el parámetro cuadrático $\alpha$ determinan juntos el tiempo necesario para que la estación de crecimiento alcance su punto medio (PM), PM $=-\beta / 2 \alpha$ (siendo $\alpha<0$ ), (de Beurs \& Henebry, 2010).

El inicio (I) y fin (F) de la estación de crecimiento se determina mediante el mejor ajuste del modelo cuadrático (de Beurs \& Henebry, 2005; de Beurs \& Henebry, 2010). El modelo cuadrático de cada estación de crecimiento fue ajustado, en múltiples tiempos de la serie temporal NDVI, mediante el coeficiente de determinación $\left(\mathrm{R}^{2}\right)$ (de Beurs \& Henebry, 2005; de Beurs \& Henebry, 2010; Brown et al., 2012).

La productividad de la vegetación estacionalmente activa de cada estación de crecimiento (Field et al., 1995; Jönsson \& Eklundh, 2006) se determinó mediante la estacionalidad integrada del NDVI (SINDVI) (Reed \& Brown, 2005), siendo:

$$
\text { SINDVI }=\Sigma \text { NDVI }_{\mathrm{ij}}
$$

En donde $i$ es el NDVI diario de la estación decrecimiento $j$. La estación de crecimiento integrada mediante el NDVI ha sido satisfactoriamente utilizada para determinar la PPN en estudios anteriormente (Tucker \& Sellers, 1986).

La base (B) es la media del NDVI del I y F de la EC: B = (NDVI I + NDVI F)/2. La amplitud (A) es la diferencia entre el NDVI del punto medio y la base: A= NDVI PM-B (Jönsson \& Eklundh, 2006). El NDVI PM es el valor del NDVI en el Punto Medio de la estación de crecimiento.

La productividad primaria neta aérea de los sistemas pastoriles (PPNA ${ }_{\mathrm{SP}}$ ) se determinó mediante el modelo de Monteith (1977):

PPNA $_{\mathrm{SP}}=\varepsilon * \Sigma(\alpha *$ SINDVI $+\beta) * \operatorname{RFA}\left(\mathrm{Mj} / \mathrm{m}^{2} . \mathrm{t}\right)$

Donde $\varepsilon$ corresponde a la eficiencia en el uso de la radiación solar (gMs/MJ) y RFA es la radiación fotosintéticamente activa. Los datos de $\varepsilon$ en el área en estudio son escasos, sin embargo puede suponerse un $\varepsilon$ constante en función de la escala del trabajo (Ruimy et al., 1994). Por cuanto para los SP en este trabajo se tomó de bibliografía el valor de $\varepsilon$ de Ruimy et al. (1994), siendo el promedio anual de pasturas perennes igual a $0.23 \mathrm{~g} \mathrm{Ms} / \mathrm{MJ}$ (Field et al., 1995). Los valores considerados de $\alpha=1.1$ y $\beta=0.055$, fueron determinados por Paruelo et al. (2004) para Sudamérica en base a la metodología propuesta por Ruimy et al. (1994). SINDVI es la estacionalidad integrada del NDVI (Reed \& Brown, 2005). La PPNA $_{S P}$ se expresó en $\mathrm{Kg} \mathrm{Ms}^{-1} \mathrm{ha}^{-1}$. 
Tabla 1. Parámetros fenológicos y PPNA de los sistemas pastoriles: I y F = fecha de inicio y fin de estación de crecimiento (día, mes, año), PM= fecha del punto medio; NDVI PM= valor del índice de vegetación en el punto medio; B = base; SINDVI= NDVI estacional integrado; ECsP= función cuadrática; $\mathrm{R}^{2}=$ coeficiente de determinación y PPNA= Productividad Primaria Neta Aérea (Kg ms/ha).

\begin{tabular}{|c|c|c|c|c|c|c|c|c|c|c|c|}
\hline & $\begin{array}{c}\text { Sistemas } \\
\text { Pastoriles }\end{array}$ & I & PM & $\mathbf{F}$ & $\begin{array}{c}\text { NDVI } \\
\text { PM }\end{array}$ & B & A & SINDVI & $\begin{array}{c}\mathrm{EC}_{\mathrm{SP}}=\alpha \mathrm{NDVI}^{2} \\
+\beta \mathrm{NDVI}+\gamma\end{array}$ & $\mathbf{R}^{2}$ & $\begin{array}{c}\text { PPNA } \\
\text { Kg Ms Ha }^{-1} \\
\end{array}$ \\
\hline \multirow[t]{4}{*}{ 2008-2009 } & SP1 & 21.01 .09 & 12.04 .09 & 29.06.09 & 0.2 & 0.04 & 0.1 & 1.5 & $\begin{array}{l}\text { ECsP1 }=-0.0025 x^{2} \\
+0.0363 x+0.0194\end{array}$ & 0.97 & 2214 \\
\hline & SP2 & 11.01 .09 & 21.01 .09 & 28.03.09 & 0.1 & 0.02 & 0.1 & 0.5 & $\begin{array}{l}\mathrm{EC}_{S P 2}=-0.0059 \mathrm{x}^{2} \\
+0.0508 \mathrm{x}-0.022\end{array}$ & 0.89 & 346 \\
\hline & SP3 & 11.01 .09 & 21.01 .09 & 28.03.09 & 0.3 & 0.13 & 0.1 & 1.7 & $\begin{array}{l}\mathrm{EC}_{\mathrm{SP} 3}=-0.0134 \mathrm{x}^{2} \\
+0.1252 \mathrm{x}-0.0055\end{array}$ & 0.87 & 1240 \\
\hline & SP4 & 05.01.09 & 28.03 .09 & 30.06 .09 & 0.1 & 0.02 & 0.1 & 2.0 & $\begin{array}{l}\text { ECsP4 }=-0.0015 x^{2} \\
+0.0322 x-0.0298\end{array}$ & 0.96 & 3361 \\
\hline \multirow[t]{4}{*}{ 2009-2010 } & SP1 & 13.12.09 & 07.04 .10 & 06.06 .10 & 0.6 & 0.25 & 0.3 & 5.9 & $\begin{array}{l}\mathrm{ECSP}_{\mathrm{SP}}=-0.0100 \mathrm{x}^{2} \\
+0.1406 \mathrm{x}+0.0971\end{array}$ & 0.98 & 9897 \\
\hline & SP2 & 21.01 .10 & 31.03 .10 & 07.06.10 & 0.6 & 0.28 & 0.3 & 6.0 & $\begin{array}{l}\text { ECsP2 }=-0.0078 x^{2} \\
+0.0785 x+0.4033\end{array}$ & 0.94 & 7649 \\
\hline & SP3 & 30.10 .09 & 07.04 .10 & 31.05 .10 & 0.6 & 0.17 & 0.4 & 6.1 & $\begin{array}{l}\text { ECsP3 }=-0.0108 x^{2} \\
+0.1756 x-0.0954\end{array}$ & 0.99 & 11230 \\
\hline & SP4 & 21.01 .10 & 07.04 .10 & 30.06 .10 & 0.5 & 0.26 & 0.3 & 5.8 & $\begin{array}{l}\mathrm{EC}_{\mathrm{SP} 4}=-0.0055 \mathrm{x}^{2} \\
+0.0606 \mathrm{x}+0.3637\end{array}$ & 0.93 & 8205 \\
\hline \multirow[t]{4}{*}{ 2010-2011 } & SP1 & 14.12 .10 & 12.03 .11 & 19.05.11 & 0.7 & 0.22 & 0.5 & 6.6 & $\begin{array}{l}\mathrm{EC}_{\mathrm{SP} 1}=-0.0064 \mathrm{x}^{2} \\
+0.1189 \mathrm{x}-0.0039\end{array}$ & 0.97 & 9225 \\
\hline & SP2 & 26.11 .10 & 28.02.11 & 06.05.11 & 0.6 & 0.20 & 0.4 & 6.8 & $\begin{array}{l}\text { ECsP2 }=-0.0075 x^{2} \\
+0.1367 x-0.0361\end{array}$ & 0.97 & 9616 \\
\hline & SP3 & 13.11 .10 & 16.01 .11 & 19.05.11 & 0.6 & 0.21 & 0.4 & 8.1 & $\begin{array}{l}\text { EC }_{S P 3}=-0.0061 x^{2} \\
+0.1088 x+0.1255\end{array}$ & 0.96 & 13600 \\
\hline & SP4 & 14.12 .10 & 28.02.11 & 29.06.11 & 0.6 & 0.18 & 0.4 & 7.6 & $\begin{array}{l}\mathrm{EC}_{\mathrm{SP} 4}=-0.0061 \mathrm{x}^{2} \\
+0.1148 \mathrm{x}+0.0475\end{array}$ & 0.96 & 13808 \\
\hline \multirow[t]{4}{*}{ 2011-2012 } & SP1 & 01.12 .11 & 31.03 .12 & 22.06 .12 & 0.5 & 0.15 & 0.4 & 7.6 & $\begin{array}{l}\text { ECsP1 }=-0.0036 x^{2} \\
+0.0761 x+0.1009\end{array}$ & 0.99 & 13935 \\
\hline & SP2 & 11.09 .11 & 31.03 .12 & 07.06.12 & 0.3 & 0.12 & 0.1 & 5.6 & $\begin{array}{l}\mathrm{EC}_{\mathrm{SP} 2}=-0.0010 \mathrm{x}^{2} \\
+0.0374 \mathrm{x}-0.0524\end{array}$ & 0.91 & 10322 \\
\hline & SP3 & 30.03 .12 & 03.05.12 & 22.06 .12 & 0.5 & 0.21 & 0.3 & 4.2 & $\begin{array}{l}\mathrm{EC}_{\mathrm{SP} 3}=-0.0099 \mathrm{x}^{2} \\
+0.1085 \mathrm{x}+0.1841\end{array}$ & 0.95 & 2992 \\
\hline & SP4 & 17.10.11 & 31.03 .12 & 22.06.12 & 0.4 & 0.09 & 0.3 & 8.1 & $\begin{array}{l}\text { ECsP4 }=-0.0022 x^{2} \\
+0.0592 x+0.0318\end{array}$ & 0.87 & 14806 \\
\hline \multirow[t]{4}{*}{ 2012-2013 } & SP1 & 03.11.12 & 01.02 .13 & 19.04.13 & 0.2 & 0.11 & 0.1 & 3.4 & $\begin{array}{l}\text { ECSP1 }_{S}=-0.0006 x^{2} \\
+0.0099 x+0.1497\end{array}$ & 0.87 & 4798 \\
\hline & SP2 & 26.01 .13 & 28.02 .13 & 05.04 .13 & 0.4 & 0.18 & 0.2 & 3.3 & $\begin{array}{l}\mathrm{EC}_{\mathrm{SP} 2}=-0.0059 \mathrm{x}^{2} \\
+0.0861 \mathrm{x}+0.0375\end{array}$ & 0.96 & 1874 \\
\hline & SP3 & 12.02 .13 & 05.03 .13 & 30.03 .13 & 0.4 & 0.28 & 0.2 & 2.9 & $\begin{array}{l}\text { ECsP3 }=-0.0133 x^{2} \\
+0.1251 x+0.1395\end{array}$ & 0.97 & 1238 \\
\hline & SP4 & 26.01 .13 & 05.03 .13 & 02.06 .13 & 0.2 & 0.12 & 0.1 & 3.9 & $\begin{array}{l}\mathrm{EC}_{\mathrm{SP} 4}=-0.0003 \mathrm{x}^{2} \\
+0.0049 \mathrm{x}+0.1586\end{array}$ & 0.92 & 4430 \\
\hline \multirow[t]{4}{*}{ 2013-2014 } & SP1 & 16.01 .14 & 15.04 .14 & 29.06.14 & 0.5 & 0.15 & 0.3 & 6.4 & $\begin{array}{l}\text { ECSP1 }=-0.0046 x^{2} \\
+0.0895 x+0.0384\end{array}$ & 0.99 & 8101 \\
\hline & SP2 & 21.01 .14 & 23.03.14 & 07.07.14 & 0.5 & 0.13 & 0.4 & 6.6 & $\begin{array}{l}\text { ECsP2 }=-0.0056 x^{2} \\
+0.1051 x+0.0251\end{array}$ & 0.97 & 9253 \\
\hline & SP3 & 10.01 .14 & 23.03.14 & 06.07 .14 & 0.6 & 0.15 & 0.4 & 7.3 & $\begin{array}{l}\text { ECSP3 }=-0.0053 x^{2} \\
+0.1097 x-0.0204\end{array}$ & 0.97 & 13300 \\
\hline & SP4 & 10.01 .14 & 23.03 .14 & 02.06 .14 & 0.5 & 0.21 & 0.3 & 5.9 & $\begin{array}{l}\text { ECsP4 }=-0.0055 x^{2} \\
+0.1011 x+0.0209\end{array}$ & 0.95 & 7460 \\
\hline
\end{tabular}


La radiación global media mensual incidente al límite de la atmósfera fue extraída de mapas de Sudamérica derivados del sensor AVHRR/NOAA19 (http://www.noaa.gov), los valores medios mensuales fueron acumulados en función de la longitud de las estaciones de crecimiento. La RFA se asume como una fracción constante del $48 \%$ de la radiación global media mensual incidente al límite de la atmósfera (Fensholt et al., 2004).

Los parámetros fenológicos Amplitud y Base serán relacionados con la PPNA mediante el coeficiente de correlación de Pearson (r) (Lee \& Wong, 2001).

El efecto estacional sobre los parámetros fenológicos y la PPNA de los sistemas pastoriles fue determinado mediante un análisis de varianza (ANAVA) en diseño completamente aleatorizado (Balzarini et al., 2008). Las variables clasificatorias fueron las estaciones de crecimiento, con las parcelas de los sistemas pastoriles como repeticiones y las variables dependientes utilizadas fueron: PPNASP, Amplitud y Base. Se verificó normalidad con ShapiroWilks modificado y homogeneidad de varianza con la prueba de Levene (Balzarini et al., 2008), no encontrándose evidencias para rechazar las hipótesis de normalidad y homogeneidad de varianzas ( $p>0.05$ ).

El modelo lineal para la observación del tratamiento $i$ en la parcela $j$, es $\mathrm{Y}_{i j}=\mu+\tau_{i}+\varepsilon$ donde $\mathrm{Y}_{i j}$ observaciones del tratamiento $i$ en la parcela $j$; $\tau_{i}$ el efecto del tratamiento $i$ y $\varepsilon$ es el término de error aleatorio asociado a la observación $\mathrm{Y}_{i j}$. La comparación de medias se realizó mediante el test de comparaciones múltiples LSD Fisher (Di Rienzo et al., 2008).

Los datos de precipitación de la serie temporal 1918 - 2014 pertenecen a la estación meteorológica de la Sociedad Rural del Noreste Santiagueño. Las anomalías fueron confeccionadas de la siguiente manera: Anomalías $=x_{i}-X_{h}$, siendo $x_{i}$ la precipitación media de un mes y $X_{h}$ la precipitación media normal o histórica (1918-2014) de ese mes (Servicio Meteorológico Nacional, 2014) (Figura 3).

\section{Resultados.}

La técnica de suavizado utilizada para la serie temporal NDVI MODIS no proveyó señales falsas con respecto a la media móvil generada (Figura 4; superiores).

En la Figura 4 (inferiores) se presentan las estaciones de crecimiento de los sistemas pastoriles (ECSP) correspondientes al período 2008-2014 derivadas de la media móvil. Las EC $\mathrm{SP}$ de los años 0809, 12-13 y en el inicio de 13-14 fueron severamente afectadas por anomalías negativas de la precipitación media mensual (Figura 4, inferiores). Las ECsp 09-10, 10-11, 11-12 y fin de 13-14, tuvieron anomalías positivas en la precipitación media mensual (Figura 4, inferiores).
La distribución de frecuencias de Inicio, Punto Medio y Fin de las estaciones de crecimiento fue confeccionada en la Tabla 4 y se presentan en la Figura 5. Los parámetros fenológicos de las EC Inicio, Punto Medio y Fin se produjeron con mayor frecuencia, respectivamente, durante enero, marzo y junio (Figura 5). Los valores del NDVI del PM oscilaron entre 0.1 y 0.7 , el menor valor se produjo en la $\mathrm{EC}_{\mathrm{SP}}$ 08-09, y el de mayor valor en la EC $\mathrm{C}_{\mathrm{SP}}$ 10-11.

Los modelos cuadráticos de las ECSP y sus respectivos coeficientes de determinación se presentan en la Tabla 4, todos los modelos tuvieron una elevada capacidad predictiva, los $\mathrm{R}^{2}$ oscilaron entre $0.87 \mathrm{y}$ 0.99 .

Se encontraron diferencias significativas ( $p=$ $0.0001 ; R^{2}=0.82$ ) en la amplitud de las estaciones de crecimiento, siendo la de mayor amplitud la EC $C_{S P} 10-$ 11 y las de menor amplitud las ECSP 08-09 y 12-13 (Tabla 2).

Tabla 2. Test de comparaciones múltiples LSD Fisher entre Amplitudes. $\mathrm{EC}_{\mathrm{SP}}=$ estación de crecimiento de sistemas pastoriles; DMS= Diferencia mínima significativa; $\mathrm{EE}=$ Error estándar; gl= grados de libertad. Medias con una letra común no son significativamente diferentes $(p>0.05)$

Test: LSD Fisher Alfa $=0.05$ DMS $=0.09484$ Error: 0.0041 gl: 18

\begin{tabular}{lllllll}
\hline ECsP & Medias & n & E.E. & & & \\
\hline EC 08-09 & 0.11 & 4 & 0.03 & A & & \\
EC 12-13 & 0.12 & 4 & 0.03 & A & & \\
EC 11-12 & 0.28 & 4 & 0.03 & & B & \\
EC 09-10 & 0.34 & 4 & 0.03 & & B & C \\
EC 13-14 & 0.35 & 4 & 0.03 & & B & C \\
EC 10-11 & 0.42 & 4 & 0.03 & & & C \\
\hline
\end{tabular}

Se encontraron diferencias significativas ( $p=$ $0.0001 ; \mathrm{R}^{2}=0.66$ ) en las bases de las estaciones de crecimiento, la $\mathrm{EC}_{\mathrm{SP}}$ 08-09 tuvo la menor base, y la EC SP $_{09}$-10 la mayor (Tabla 3).

Tabla 3. Test de comparaciones múltiples LSD Fisher entre Bases. $\mathrm{EC}_{\mathrm{SP}}=$ estación de crecimiento de sistemas pastoriles; DMS= Diferencia mínima significativa; EE= Error estándar; gl= grados de libertad. Medias con una letra común no son significativamente diferentes $(p>0.05)$

Test: LSD Fisher Alfa $=0.05$ DMS $=0.12747$ Error: 0.0025 gl: 18

\begin{tabular}{lcccccc}
\hline EC & Medias & n & E.E. & & & \\
\hline EC 08-09 & 0.05 & 4 & 0.02 & A & & \\
EC 11-12 & 0.14 & 4 & 0.02 & & B & \\
EC 13-14 & 0.16 & 4 & 0.02 & & B & \\
EC 12-13 & 0.17 & 4 & 0.02 & B & C \\
EC 10-11 & 0.20 & 4 & 0.02 & & B & C \\
EC 09-10 & 0.24 & 4 & 0.02 & & & C \\
\hline
\end{tabular}


Se encontraron diferencias significativas $(p=$ 0.0003; $\mathrm{R}^{2}=0.71$ ) en la PPNA de las estaciones de crecimiento, las $\mathrm{EC}_{\mathrm{SP}}$ de menor productividad en el período fueron las de 08-09 y 12-13, no habiendo diferencias significativas entre el resto de las $\mathrm{EC}_{\mathrm{SP}}$ (Tabla 4).

Tabla 4. Test de comparaciones múltiples LSD Fisher entre PPNA. $\mathrm{EC}_{\mathrm{SP}}=$ estación de crecimiento de sistemas pastoriles; DMS $=$ Diferencia mínima significativa; EE= Error estándar; gl= grados de libertad. Medias con una letra común no son significativamente diferentes $(p>0.05)$

Test: LSD Fisher Alfa= 0.05 DMS= 0.38326 Error: 0.0666 gl: 18

\begin{tabular}{ccclll}
\hline ECSP & Medias & n & E.E. & & \\
\hline EC 08-09 & 1790.20 & 4 & 1435.89 & A & \\
EC 12-13 & 3085.22 & 4 & 1435.89 & A & \\
EC 09-10 & 9245.23 & 4 & 1435.89 & & B \\
EC 13-14 & 9528.51 & 4 & 1435.89 & & B \\
EC 11-12 & 10514.00 & 4 & 1435.89 & & B \\
EC 10-11 & 11562.31 & 4 & 1435.89 & & B \\
\hline
\end{tabular}

La amplitud y la PPNA de los sistemas pastoriles de Panicum maximun presentaron relaciones directa significativas $\left(p<0.01 ; \mathrm{R}^{2}=0.50\right)$. A su vez, no se encontraron relaciones significativas entre la base y la PPNA $(p>0.05)$

\section{Discusión.}

La mayor frecuencia del inicio de las estaciones en enero evidencia la marcada dependencia de los sistemas pastoriles de Panicum maximun a las precipitaciones estivales (Figura 3). En ambientes semiáridos la vegetación responde de manera clara e inmediata ante las precipitaciones, siendo evidenciados por picos de NDVI (Nicholson et al., 1990; Wang et al., 2003). En este sentido, Zerda \& Tiedemann (2010) al caracterizar la dinámica interanual y mensual del NDVI VGT del bosque y pastizal natural de Santiago del Estero en el período 1999-2002, determinaron una respuesta exponencial del NDVI del pastizal (0.4 NDVI/día) debido al inicio de las precipitaciones. Los inicios de la estación de crecimiento con menor frecuencia (octubre, noviembre y diciembre), se relacionan con el inicio temprano, poco frecuente, de la estación lluviosa. A su vez, la disponibilidad de humedad y temperatura favorece la persistencia del rebrote térmico de los pastizales (Renolfi, 1988). Adicionalmente, Pérez (1992) determinó que la fase vegetativa de las gramíneas en el Chaco semiárido se concentra entre septiembre y enero y se relaciona ampliamente con la estación lluviosa.

La mayor frecuencia de PM en marzo y abril se relaciona con el incremento del porcentaje de efectividad de las precipitaciones en toda el área de estudio y de la región en general (Torres Bruchman,
1981). Estos resultados contrastan con los de León (2004) quien a partir de ensayos regionales de gramíneas megatérmicas comunica que la productividad estacional del Panicum maximun en Santiago del Estero, se produce en un $70 \%$ durante el verano. Resultados similares obtuvieron Chiossone \& Vicini (2012) quienes al evaluar el comportamiento del Panicum maximun en la Provincia del Chaco en el período 2008-2011, determinaron que la mayor producción de materia seca se produjo en verano: el $35.5 \%$ en noviembre-diciembre y el $44.4 \%$ en enerofebrero. Sin embargo los resultados obtenidos en este trabajo son consistentes, fundamentalmente porque la metodología de análisis utilizada es más adecuada para hacer inferencias a escala regional que la utilizada por los autores antes mencionados. Adicionalmente, Tiedemann (2011) y Tiedemann et al. (2012) al analizar la variación de los parámetros fenológicos del bosque y pastizal natural en un período húmedo y otro seco en la Provincia de Santiago del Estero, mediantes series temporales NDVI VGT, determinaron que los puntos medios y NDVI máximos de ambas coberturas se producen en otoño (marzo) en ambos períodos.

El fin de las estaciones de crecimiento se produjo con mayor frecuencia en junio y en menor medida en mayo (Figura 5), esta respuesta está relacionada con la marcada estacionalidad de las forrajeras subtropicales y de la vegetación natural de la región en general. Chiossone \& Vicini (2012) obtuvieron similares resultados, ellos comunicaron que el fin de la oferta forrajera del Panicum maximun en la Provincia del Chaco en el período 2008-2011 se produjo en mayojunio. Cornacchione \& Molina (2008) al determinar fechas de siembra óptimas del Panicum maximun comunicaron que la productividad fue severamente afectada por las bajas temperaturas y heladas tempranas. Tiedemann (2011) determinó que el fin de la estación de crecimiento del pastizal natural en un período húmedo se producía a partir de mayo, mientras que en un período con sequía se producía a partir de abril. La finalización de estaciones en otoño concuerdan con los obtenidos por de León (2004) quien comunica que el $20 \%$ de producción del Panicum maximun se produce al finalizar la estación de crecimiento en otoño. La finalización de las estaciones de crecimiento en marzo y abril fueron menos frecuentes (Figura 5), éstas coinciden con las estaciones de crecimiento afectadas por sequía. Debe considerarse que el estrés hídrico reduce la tasa de fotosíntesis (Peters \& Eve, 1995; Weiss et al., 2004) produce alta mortandad de hojas y plantas (Renolfi, 1988; Karnieli et al., 2002; Weiss et al., 2004) e induce a la vegetación a tomar estrategias extremas como la caída de hojas que afectan directamente los valores del NDVI (Ray, 1995; Karnieli et al., 2002). 

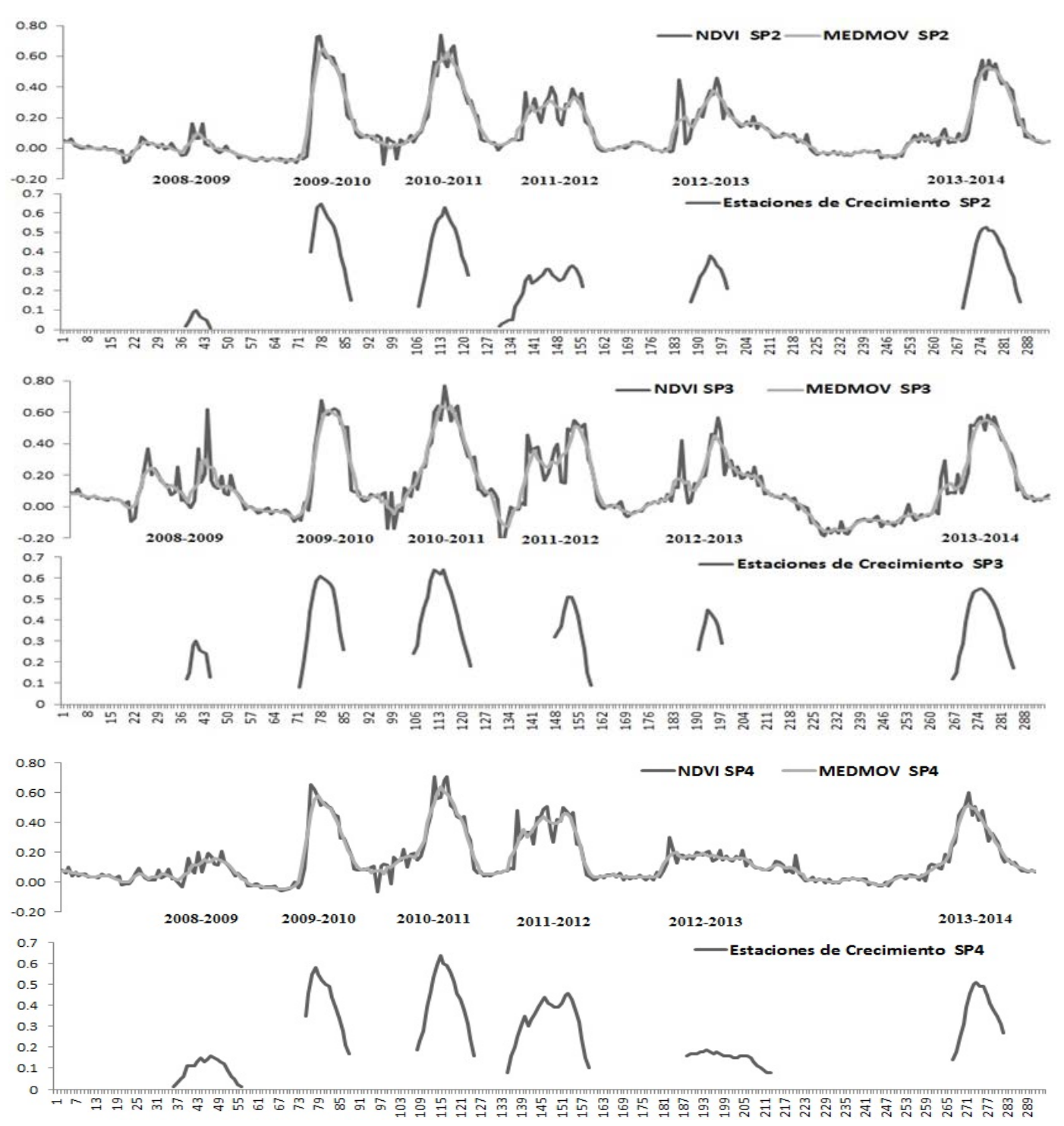

Figura 4. Figuras superiores: Serie temporal original NDVI MODIS 2008-2014 (línea negra) y serie temporal suavizada o media móvil (línea gris) de los sistemas pastoriles SP1, SP2, SP3 y SP4. Figuras inferiores: Estaciones de crecimiento de los SP1, SP2, SP3 y SP4 derivadas de la media móvil.

La amplitud de las estaciones de crecimiento es un parámetro dependiente del valor de la base y del NDVI del Punto Medio (Jönsson \& Eklundh, 2004; Heumann et al., 2007), por cuanto las EC $\mathrm{SP}_{\mathrm{S}}$ afectadas por anomalías negativas de precipitación son las que tuvieron menores valores de NDVI PM y base y por ende las menores amplitudes. De manera inversa las EC $C_{S P}$ con anomalías positivas de precipitación tuvieron los valores más elevados de NDVI PM y base y por ende mayores amplitudes. Tiedemann (2011) al analizar la dinámica estacional del NDVI VGT del pastizal nativo (Elionorus sp.) en Santiago del Estero, determinó que la amplitud de esa cobertura era menor en el período húmedo y mayor en el seco, y debido a que el NDVI del PM fue similar en ambos periodo, concluyó que la amplitud del pastizal estuvo influenciada por los valores más elevados de la base durante el periodo húmedo. En su relación con la productividad estacional, las EC $\mathrm{C}_{\mathrm{SP}}$ con menor amplitud son las que tuvieron menor PPNA, ambos parámetros fueron severamente afectados por las anomalías negativas de precipitación. Debido a las significativas relaciones directas encontradas entre la amplitud y la PPNA, podríamos considerar a la amplitud de las EC $_{S P}$ un rápido estimador de la PPNA estacional.

La base es un parámetro fenológico dependiente del NDVI del inicio y fin de las estaciones de crecimiento (Jönsson \& Eklundh, 2004; Heumann et al., 2007). La EC SP 08-09, tuvo una base extremadamente baja, mientras que la EC $\mathrm{C}_{S P} 12-13$ tuvo una base moderada, ambas estaciones fueron 


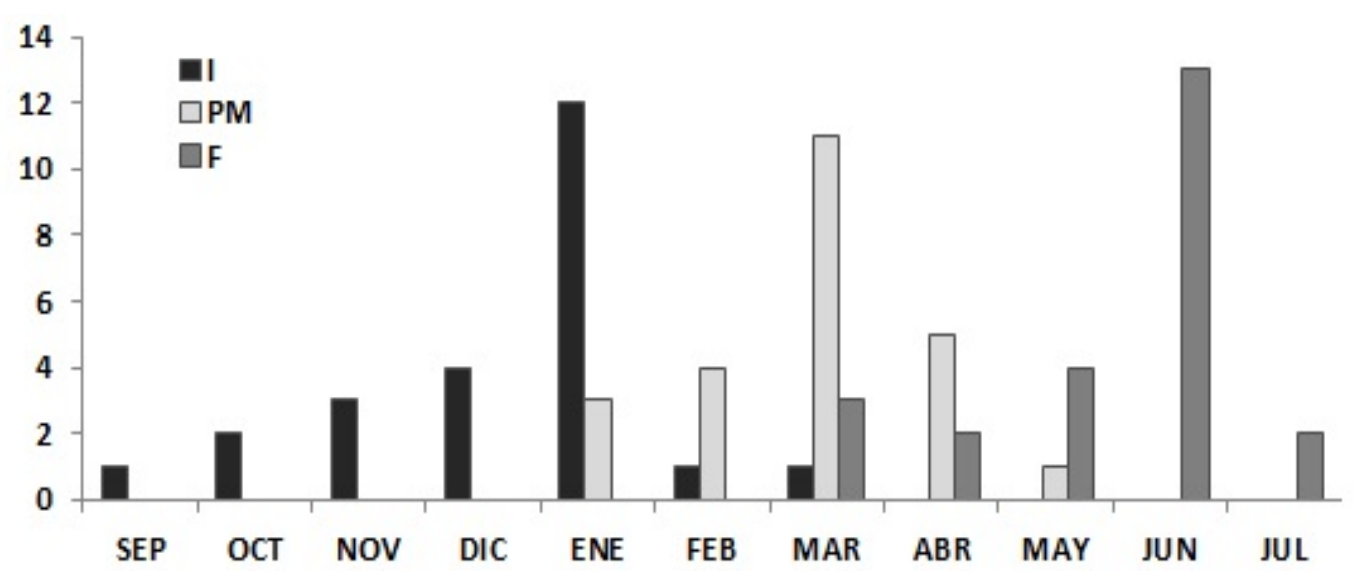

Figura 5. Distribución de trecuencias de las techas de Inicio (I), Punto Medio (PM) y Fin (F) de las estaciones de crecimientos de los sistemas pastoriles en el período 2008-2014.

severamente afectadas por la sequía regional, y fueron las de menor PPNA. La EC $\mathrm{sP}$ 09-10 tuvo la mayor base y una elevada PPNA. Debido a que no se encontraron relaciones entre la base y la PPNA, podemos considerar que la base y por ende el NDVI de inicio y fin de estación de crecimiento no incidieron en la PPNA estacional de los sistemas pastoriles.

La PPNA de las ECsP fue severamente afectada por la sequía regional. El Panicum maximun es muy sensible a las sequías (de León, 2004). La falta de precipitaciones o sequía reduce el crecimiento de la vegetación e induce a un decrecimiento en la capacidad fotosintética que es detectado y cuantificado mediante el NDVI y anomalías del NDVI (Tucker \& Choudhury, 1987). De acuerdo a Rigge et al. (2013) el déficit de precipitaciones y la elevada variabilidad interanual limitan la Productividad Neta del Ecosistema, por cuanto las pasturas se vuelven una fuente neta de carbono durante años con sequía. La PPNA de las EC SP afectadas con anomalías negativas de precipitación (08-09 y 12-13) fue un 78,7 \% menor que la PPNA de las $\mathrm{EC}_{S P}$ con anomalías positivas de precipitación media. Similares resultados obtuvieron Chiossone \& Vicini (2012) al comunicar que la sequía redujo la productividad del Panicum maximun en un $70 \%$ en la Provincia del Chaco. Por el contrario, las $\mathrm{EC}_{\mathrm{SP}}$ con anomalías positivas de precipitaciones tuvieron similar PPNA, de acuerdo a Rigge et al. (2013) las pasturas son sumideros de carbono cuando presentan disponibilidad de precipitaciones y manejo adecuado.

Los valores de PPNA estimados en este trabajo se encuentran dentro del rango de PPNA determinados por la red de ensayos locales. Kunst et al. (2001) comunica que la productividad acumulada del Panicum maximun oscila entre los 6000 y $14000 \mathrm{~kg}$ $\mathrm{ms} \mathrm{ha}^{-1}$. A su vez, de León (2004) en el marco del proyecto ganadero regional del Instituto Nacional de Tecnología Agropecuaria (INTA) (www.inta.gob.ar) comunica que la productividad de biomasa forrajera del Panicum maximun cv Gatton en Santiago del Estero con precipitaciones de $400 \mathrm{~mm}$ es de $4000 \mathrm{~kg}$ $\mathrm{ms} \mathrm{ha}^{-1}$, mientras que con precipitaciones de $800 \mathrm{~mm}$, la productividad ronda los $7500 \mathrm{~kg} \mathrm{~ms} \mathrm{ha-1}$. A su vez, Cornacchione \& Mijoevich (2011) responsables del módulo NOA de la red de evaluación de especies megatérmicas del INTA, comunicaron que la producción de materia seca acumulada del Panicum maximun cv Gatton en las estaciones de crecimiento 06-07 y 07-09 osciló entre los 11000 y 12000 kg ms ha $^{-1}$, la productividad en la estación 08-09 presentó valores entre los 4000 y $5000 \mathrm{~kg} \mathrm{~ms} \mathrm{ha}^{-1}$, mientras que en la estación de crecimiento 09-10, la productividad osciló entre los 14000 y 15000 kg ms ha-1. Cornacchione \& Molina (2008) con el fin de determinar fechas óptimas de siembra y la productividad del Panicum maximun cV Gatton, realizaron la implantación en diferentes fechas de siembra, tres siembras entre noviembre y diciembre y dos siembras en febrero. Ellos comunicaron que las fechas tempranas fueron las más efectivas y que la PPNA de las tres fechas no tuvieron diferencias significativas, siendo estas: $9330 \mathrm{~kg} \mathrm{~ms} \mathrm{ha}{ }^{-1} ; 10665 \mathrm{~kg}$ $\mathrm{ms} \mathrm{ha}^{-1}$ y $8063 \mathrm{~kg} \mathrm{~ms} \mathrm{ha}{ }^{-1}$.

La longitud de las estaciones de crecimiento es dependiente de las fechas de inicio y fin de la estación de crecimiento (Jönsson \& Eklundh, 2004; Heumann et al., 2007). Las $\mathrm{EC}_{\mathrm{SP}}$ con menor longitud tuvieron menor PPNA, mientras que las de mayor longitud tuvieron mayor PPNA. La longitud incide de manera directa en la PPN del ecosistema (Field et al., 1995; White et al., 1997; Reed \& Brown, 2005; Arora \& Boer, 2005; Jönsson \& Eklundh, 2006; Cleland et al., 2007; Chandola et al., 2010).

\section{Conclusiones.}

La serie temporal NDVI derivada del sensor MODIS, permitió determinar los parámetros fenológicos, modelar las estaciones de crecimiento y a 
partir de ellas cuantificar la PPNA estacional de los sistemas pastoriles del Dpto. Moreno, Santiago del Estero, Argentina en el período 2008-2014.

Los parámetros fenológicos (amplitud y base) y la PPNA de las estaciones de crecimiento de los sistemas pastoriles Panicum maximun fueron significativamente afectados por las anomalías negativas de precipitación media estacional.

Los parámetros fenológicos longitud y amplitud de las estaciones de crecimiento están directamente relacionadas con la PPNA de los sistemas pastoriles de Panicum maximun.

La PPNA estimada mediante sensores remotos de los sistemas pastoriles de Panicum maximun oscila dentro de los valores de productividad de materia seca determinados por las redes de ensayos locales a escala uno a uno en el Dpto. Moreno, Santiago del Estero, Argentina.

La determinación de los parámetros fenológicos y PPNA de los sistemas pastoriles derivados de series temporales NDVI MODIS, se transforma en una invalorable herramienta que dispone la comunidad agropecuaria, por un lado para determinar de manera eficiente la receptividad de los potreros y por el otro para prevenir y/o mitigar los efectos de adversidades climáticas extremas como sequías.

Los resultados de este trabajo podrían considerarse un punto de partida en el avance del conocimiento, a escala local, sobre la fenología y productividad de los sistemas pastoriles de Panicum maximun del Chaco semiárido, derivada de sensores remotos.

\section{Agradecimientos.}

- Al Dr. Hugo R. Zerda. Director del proyecto que enmarca este trabajo, denominado - Monitoreo satelital de la distribución espacial y temporal de las coberturas y usos de la tierra de la provincia de Santiago del Estero-, CICyT-UNSE No 23/B090.

- A la Dra. Liliana Diodato. Directora del Instituto de Protección Vegetal - FCF - UNSE.

- Al Profesor Ramón Ledesma - FCF - UNSE, por la revisión del summary.

\section{Literatura citada.}

Abril A. \& Bucher E.H. 2001.Overgrazing and soil carbon dynamics in the western Chaco of Argentina. Applied Soil Ecology 16: 243-249.

Anriquez A., Albanesi A., Kunst C., Ledesma R., Lopez., Rodriguez Torresi A. \& Godoy J. 2005. Rolado de fachinales y calidad de suelos en el chaco occidental, Argentina. CI. Suelo, 23: 145-157.

Argentina. 2013. Programa de Competitividad Conglomerado Bovino de Santiago del Estero. Secretaría de Política Económica del Ministerio de Economía y Finanzas Públicas de la Nación, 42p.

Arora V. K \& Boer G.J. 2005. A parameterization of leaf phenology for the terrestrial ecosystem component of climate models, Global Change Biology, 11:39-59.

Asrar G., Funchs M., Kanemasu E.T. \& Hatfield J.L. 1984. Estimating absorbed photosynthetic radiation and leaf area index from spectral reflectance in wheat. Agronomy Journal, 76:300-306.

Atkinson P.M., Jeganathan C., Dash J. \& Atzberger C.2012. Inter-comparison of four models for smoothing satellite sensor time-series data to estimate vegetation phenology. Remote Sensing of Environment 123: 400417.

Balzarini M.G., Gonzalez L., Tablada M., Casanoves F., Di Rienzo J.A., Robledo C.W. 2008. Manual del Usuario, Editorial Brujas, Córdoba, Argentina.

Becker-Reshef I., Justice C., Sullivan M., Vermote M., Tucker C., Anyamba A., Small J., Pak E., Masuoka E., Schmaltz J., Hansen M., Pittman K., Birkett C., Williams D., Reynolds C. \& Doorn B. 2010. Monitoring Global Croplands with Coarse Resolution Earth Observations: The Global Agriculture Monitoring (GLAM) Project. Remote Sensing 2:1589-1609.

Boletta P., Ravelo C.A., Planchuelo A.M. \& Grilli M. 2006. Assessing deforestation in the Argentine Chaco. Forest Ecology and Management 228:108-114.

Bradley B.A., Jacob R.W., Hermance J.F. \& Mustard J.F. 2007. A curve fitting procedure to derive inter-annual phenologies from time series of noisy satellite NDVI data. Remote Sensing of Environment 106:137-145.

Brown M.E., de Beurs K.M. \& Marshall M. 2012. Global phenological response to climate change in crop areas using satellite remote sensing of vegetation, humidity and temperature over 26 years. Remote Sensing of Environment 126:174-183.

Chandola V., Dafeng Hui., Lianhong Gu., Bhaduri B. \& Vatsavai R.R. 2010.

Using Time Series Segmentation for Deriving Vegetation Phenology Indices from MODIS NDVI Data. Data Mining Workshops (ICDMW), IEEE International Conference.

DOI 10.1109/ICDMW.2010.143

Chiossone J. \& Vicini R. 2012. Producción de Materia Seca De Gatton Panic en el Dpto. Almirante Brown en diferentes Sistemas de Manejo. Ed. INTA EEA Sáenz Peña. Disponible en: http://inta.gob.ar/documentos/produccion-de-materiaseca-de-gatton-panic-en-el-dpto.-almirante-brown-endiferentes-sistemas-de-manejo/

Cleland E., Chuine I., Menzel A., Mooney H.A. \& Schwartz M.D. 2007. Shifting plant phenology in response to global change. TRENDS in Ecology and Evolution 22:357-364

Cornacchione M. \& Molina J.P. 2008. Implantación de gramíneas subtropicales según fechas de siembra y acumulación y componentes de la materia seca. Revista Argentina de Producción Animal 28:349-543.

\& Mijoevich L. 2011. Ensayo comparativo de rendimiento de gramíneas megatérmicas. Ciclo de evaluación 2006-2010. INTA EEASE. Red de evaluación de especies megatérmicas del Instituto Nacional de Tecnología Agropecuaria. Disponible en: http://inta.gob.ar/documentos/red-de-evaluacion-deespecies-megatermicas.-informe-resumido-de-proyectociclos-2006-2010/

de Beurs K. M \& Henebry G. M. 2005. A statistical framework for the analysis of long image time series. International Journal of Remote Sensing 26:1551-1573. 
\& Henebry G. M. 2010. Spatio-Temporal Statistical Methods for Modelling Land Surface Phenology En: Phenological Research, I.L. Hudson, M.R. Keatley (eds.).

de León M. 2004. Las pasturas subtropicales en la región semiárida central del país. Informe Técnico $\mathrm{N}^{\mathrm{o}} 1$, Instituto Nacional de Tecnología Agropecuaria, Centro Regional Córdoba, ISSN 1668-2890. Disponible en: http://www.produccion-

animal.com.ar/produccion_y_manejo_pasturas/pasturas_ cultivadas_megatermicas/55ampliando_frontera_ganadera.pdf.

Di Bella C., Faivre R., Ruget F., Seguin B., Guerif M., Combal B., Weiss M. \& Rebella C. 2004. Remote sensing capabilities to estimate pasture production in France, Int. J. Remote Sensing 23:5359-5372.

Di Rienzo J.A., Casanoves F., Balzarini M.G., Gonzalez L., Tablada M. \& Robledo C.W. 2008. InfoStat, versión 2008, Grupo InfoStat, FCA, Universidad Nacional de Córdoba, Argentina.

Eastman R., McKendry J.E \& Fulk M.A. 1995. Change and times series analysis. Explorations in Geographic Information Systems Technology. UNITAR Second Edition, Palais des Nations, Geneva, Suiza, 116p. 2009. IDRISI Taiga 16.03. The IDRISI Project, University's George Perkins Marsh, Institute at Clark University.

Goetz S.J. \& Prince S.D. 1996. Remote sensing of net primary production in boreal forest stands. Agricultural and Forest Meteorology 78: 149-179.

, Prince S.D., Goward S.N., Thawley M.M., Small J. \& Johnston A. 1999. Mapping net primary production and related biophysical variabkes with remote sensing: Application to the BOREAS region. Journal of Geogphysical Research, 104:27.719-27.734.

Fensholt R. 2003. Assessment of Primary Production in Semi-arid Environment from Satellite Data. Exploiting capabilities of new sensors. Ph. D. dissertation, Institute of Geography, University of Copenhagen, Copenhagen K, Denmark, 87p.

Sandholt I. \& Schultz Rasmussen M. 2004 Evaluation of MODIS LAI, fAPAR and the relation between fAPAR and NDVI in a semi-arid environment using in situ measurements. Remote Sensing of Environment 91:490-507.

, Sandholt I., Schultz Rasmussen M., Stisen S. \& Diouf A.2006. Evaluation of satellite based primary production modelling in the semi-arid Sahel. Remote Sensing of Environment 105:173-188.

Field C.B., Randerson J.T. \& Malmstriik C.M. 1995. Global Net Primary Production: Combining Ecology and Remote Sensing. Remote Sensing Environment 51: 7488.

Heumann B.W., Seaquist J.W., Eklundh L. \& Jonsson P. 2007. AVHRR derived phenological change in the Sahel and Soudan, Africa, 1982-2005. Remote Sensing of Environment 108:385-392.

Hicke A. J., Asner P. G., Randerson T.J., Tucker C., Los S., Birdsey R; Jenkins J; Field C. \& Holland E. 2002. Satellite-derived increases in net primary productivity across North America, 1982-1998. Geophysical Research Letters, 29: 69-1-69-4.

Ho J., Ryu D. \& Costelloe J. 2011. Comparative Analysis of NDVI and Microwave-Derived Soil Moisture for
Determining Surface Water in the Cooper Creek Catchment, 19th International Congress on Modelling and Simulation, Perth, Australia, 12-16 December 2011. Disponible http://mssanz.org.au/modsim2011/E4/ho.pdf

Jolly W.M., Nemani R. \& Running S.W. 2005. A generalized, bioclimatic index to predict foliar phenology in response to climate, Global Change Biology, 11:619-632.

Jönson P. \& Eklundh L. 2004. TIMESAT- a program for analyzing time-series of satellite sensor data. Computers \& Geosciences 30:833-845.

2006. TimesatGUI. Users guide for version TIMESAT 2.2, $17 \mathrm{pp}$.

Karnieli A., Gabai A., Ichoku C., Zaady E. \& Shachak M. 2002. Temporal dynamics of soil and vegetation spectral responses in a semi-arid environment. International J. of Remote Sensing, 19:4073-4087.

Kunst C., Cornacchione M.V., Gelid L. \& Godoy J. 2001. Aumento de forraje. Manejo desmonte y postdesmonte. Persistencia de pasturas subtropicales. En: Jornada sobre intensificación de la producción ganadera y sustentabilidad de recursos naturales en Santiago del Estero. Junio de 2001, Quimilí, Santiago del Estero, Argentina, 27pp.

., Ledesma R., Basan M., Angella G., Prieto D. \& Godoy J. 2003. Rolado de fachinales e infiltración de agua en el suelo en el Chaco occidental argentino. Revista de Investigaciones Agropecuarias 32: 105-122. ., Monti E; Perez H. \& Godoy J. 2006. Assessment of the rangelands of southwestern Santiago del Estero, Argentina, for grazing management and research. Journal of Environmental Management 80:248-265. , Ledesma R \& Navall M. 2008. RBI: Rolado selectivo de baja intensidad, $139 \mathrm{p}$, en ediciones INTA disponible en: http://inta.gob.ar/documentos/rbi-roladoselectivo-de-baja-intensidad/

., Ledesma R; Bravo S; Albanesi A. \& Godiy J. 2012. Disrupting woody states in the Chaco region (Argentina): responses to combined disturbance treatments. Ecological Engineering 42:42-53.

Ledesma R. 2006. Desarrollo de sistemas ganaderos: Una alternativa de manejo en ecosistemas degradados del Chaco semiárido, Santiago del Estero, Argentina. Universidad Nacional de Santiago del Estero, Tesis de maestria, $134 \mathrm{p}$.

Lee J. \& Wong D.W. 2001. Statistical Analysis with ArcView GIS. John Wiley \& Sons, INC. New York, USA, 2001. pp. 192.

Monteith J.L. 1977. Climate and the efficiency of crop production in Britain. Philosophical Transactions of the Royal Society of London B 281:277-294.

Morello J. \& Saravia Toledo C. 1959. El bosque Chaqueño II. La ganadería y el bosque en el oriente de Salta. Rev. Agron. Noroeste Arg. 3:209-258.

Nicholson S.E., Davenport L.M., \& Malo A.R. 1990. A comparison of the vegetation response to rainfall in the Shael and East Africa, using Normalized Difference Vegetation Index from NOAA AVHRR, Climatic Change 17:209- 241.

Nouvellon Y., Seen D. L., Rambal S., Begé A., Moran M., Kerr Y. \& Qi J. 2000. Time Course of Radiation Use Efficiency in a Shortgrass Ecosystem: Consequences for 
Remotely Sensed Estimation of Primary Production. Remote Sens. Environ. 71:43-55.

Paruelo J. M., Golluscio R.A., Guerschman J.P., Cesa A., Jouvé V. \& Garbulsky M.F. 2004. Regional scale relationships between ecosystem structure and functioning. The case of the Patagonian steppes. Global Ecology and Biogeography 13: 385- 395.

Pérez H.E. 1992. Las pasturas cultivadas en la EEA INTA Santiago del Estero. En: Jornadas de actualización técnica en producción bovina en áreas de secano de Santiago del Estero, INTA, 162 p, 23, 24 y 25 de abril, Santiago del Estero, Argentina.

Peters A.J. \& Eve M.D. 1995. Satellite monitoring of desert plant community response to moisture availability. Environmental Monitoring and Assessment 37:273-287.

Ray T.W. 1995. Remote Monitoring of Land Degradation in Arid/Demiarid Regions. Thesis by Doctor of Phillosophy, California Institute of tecnology, Pasadena, California.

Reed B.C., White M. \& Brown J.F. 2003. Remote Sensing Phenology. En: Phenology: An Integrative Enviromental Science. Schwartz M. D (Ed), Kluwer Academic Publisher Netherlands, $557 \mathrm{p}$. \& Brown J.F. 2005. Trend Analysis of Timeseries Phenology Derived from Satellite Data, IEEE Xplore Librery digital; Disponible en: http://ieeexplore.ieee.org/stamp/stamp.jsp?arnumber=01 469863

Renolfi R.F. 1988. Producción y manejo de forrajeras introducidas y nativas en el Chaco Semiárido. En: Curso taller Internacional, Forrajeras y cultivos adecuados para la Región Chaqueña Semiárida. Editor: Red de cooperación técnica en uso de recursos naturales en la región chaqueña semiárida (Argentina, Bolivia, Paraguay), La Rioja, Argentina, 59-69 pp.

. 1992. Los pastizales de la Región Chaqueña. En: Jornadas de actualización técnica en producción bovina en áreas de secano de Santiago del Estero. Editor: INTA EEA Santiago del Estero, Argentina, 17$26 \mathrm{pp}$.

Rigge R., Wylieb B., Zhangc L. \& Boyted S.P. 2013 Influence of management and precipitation on carbon fluxes in great plains grasslands. Ecological Indicators 34:590-599.

Rouse J.W., Haas R.H., Schell J.A. \& Deering D.W. 1973. Monitoring vegetation systems in the Great plains with ERTS, Third RTS Symposium, NASA SP-351 I, 309317.

Ruimy A., Saugier B. \& Dedieu D. 1994. Methodology for the estimation of terrestrial net primary production from remotely sensed data. Journal of Geophysical Research 99: 5263-5283.

Secretaría de Ambiente y Desarrollo Sustentable de la Nación (SAyDS). 2004. Parque Chaqueño. En: Atlas de los Bosques Nativos Argentinos, Proyecto Bosques Nativos y Áreas protegidas, Argentina, 244 pp.
2007. Informe Regional Parque Chaqueño. En: Primer inventario nacional de bosques nativos, Proyecto Bosques Nativos y Áreas protegidas, Argentina, 114p.

Servicio Meteorológico Nacional. 2014. Servicios climáticos, mapas de anomalías de precipitación acumulada. Disponible En: http://www.smn.gov.ar/serviciosclimaticos/?mod=elclim a\&id $=72$

Smit G.N. 2005. Tree thinning as an option to increase herbaceous yield of an encroached semi-arid savanna in South Africa. BMC Ecology 5:4 doi:10.1186/14726785-5-4.

Tan B., Morisette J.T; Wolfe R. E; Gao F; Ederer G. A; Nightingale J. \&

Pedelty J.A. 2011. An Enhanced TIMESAT Algorithm for Estimating Vegetation Phenology Metrics From MODIS Data. IEEE Journal of Selected Topics in Applied Earth Observations and Remote Sensing, 4:361371.

Tiedemann J.L. 2011. Dinámica espacial y temporal del Índice de Vegetacion de Diferencia Normalizada en Santiago del Estero. Tesis Doctoral, Escuela de Graduados Facultad de Ciencias Agropecuarias, Universidad Nacional de Córdoba, Argentina, 157p.

, Zerda H.R., Grilli M. \& Ravelo A. C. 2012. Variabilidad fenológica del bosque y del pastizal nativo en el Chaco Semiárido de la Provincia de Santiago del Estero, Argentina. Ambiência 8:47-60.

Torres Bruchmann E. 1981. Climatología General y Agrícola de la Provincia de Santiago del Estero. Universidad Nacional de Tucumán, Tucumán, Argentina, 199 pp.

Tucker J.C. \& Sellers P.J. 1986. Satellite remote sensing of primary production. Int. J. Remote Sensing, 11:13951416.

\& Choudhury J.B. 1987. Satellite remote sensing of drought conditions. Remote Sensing of Environment 23: 243-251.

Wang J., Price K.P. \& Rich P.M. 2003. Temporal responses of NDVI to precipitation and temperature in the central Great Plains, USA. Int. J. Remote Sensing, 11:23452364.

Weiss J.L., Gutzler D.S., Allerd Coonrod J.E. \& Dahm C.N. 2004. Long-term vegetation monitoring with NDVI in a diverse semi-arid setting, central New Mexico, USA. Journal of Arid Environments 58:248-271.

White A.M., Thornton E.P. \& Running W.S. 1997. A continental phenology model for monitoring vegetation responses to interannual climatic variability. Global Biogeochemical Cycles, 2:217-234.

Zerda H. R. \& Tiedemann J.L. 2010. Dinámica temporal del NDVI del bosque y pastizal natural en el Chaco Seco de la Provincia de Santiago del Estero, Argentina. Ambiência, 6:13-24.

\footnotetext{
${ }^{1}$ Instituto de Protección Vegetal, Facultad de Ciencias Forestales - Universidad nacional de Santiago del Estero, Av. Belgrano Sur, 1912, (CP 4200) Capital, Santiago del Estero, Argentina.
} 